\title{
Well quasi-order in combinatorics: embeddings and homomorphisms
}

\author{
S. Huczynska and N. Ruškuc
}

\begin{abstract}
The notion of well quasi-order (wqo) from the theory of ordered sets often arises naturally in contexts where one deals with infinite collections of structures which can somehow be compared, and it then represents a useful discriminator between 'tame' and 'wild' such classes. In this article we survey such situations within combinatorics, and attempt to identify promising directions for further research. We argue that these are intimately linked with a more systematic and detailed study of homomorphisms in combinatorics.
\end{abstract}

\section{Introduction}

In combinatorics, indeed in many areas of mathematics, one is often concerned with classes of structures that are somehow being compared, e.g. in terms of inclusion or homomorphic images. In such situations one is naturally led to consider downward closed collections of such structures under the chosen orderings. The notion of partial well order (pwo), or its mild generalisation well quasi-order (wqo), can then serve to distinguish between the 'tame' and 'wild' such classes. In this article we will survey the guises in which wqo has made an appearance in different branches of combinatorics, and try to indicate routes for further development which in our opinion will be potentially important and fruitful.

The aim of this article is to identify major general directions in which wqo has been deployed within combinatorics, rather than to provide an exhaustive survey of all the specific results and publications within the topics touched upon. In this section we introduce the notion of wqo, and present what is arguably the most important foundational result, Higman's Theorem. In Section 2 we attempt a broad-brush picture of wqo in combinatorics, linking it to the notion of homomorphism and its different specialised types. The central Sections 3-5 present three 'case studies' words, graphs and permutations - where wqo has been investigated, and draw attention to specific instances of patterns and phenomena already outlined in Section 2. Finally, in Section 6, we reinforce the homomorphism view-point, and explore possible future developments from this angle.

A quasi-order (qo) is any binary relation which is reflexive $(x \leq x$ for all $x$ ) and transitive $(x \leq y \leq z$ implies $x \leq z)$. If our quasi-order 
is also anti-symmetric $(x \leq y \leq x$ implies $x=y)$, it is called a partial $\operatorname{order}(p o)$. We will write $x<y$ to denote $x \leq y$ and $y \not \leq x$. Kruskal [42] gives the rule-of-thumb that it is easier to work with partial order than quasi-order "at a casual level", but that "in advanced work, the reverse is true". In fact, many natural "comparison relations", such as via embeddings or homomorphic images, are genuine quasi-orders, and become orders only when we restrict attention to finite structures. The close connection between the two notions is encapsulated by the fact that any qo gives rise to a po on the equivalence classes $(x \equiv y$ if $x \leq y \leq x)$.

A well quasi-order (wqo) is a qo which is

- well-founded: every strictly decreasing sequence is finite; and

- has no infinite antichain: every set of pairwise incomparable elements is finite.

There are various widely-used equivalent formulations of what it means to be wqo, such as:

Theorem 1.1 The following are equivalent for a quasi-order $\leq$ on a set $X$ :

(i) $\leq$ is a well quasi-order;

(ii) if $x_{0}, x_{1}, \ldots \in X$ then there are $i<j$ with $x_{i} \leq x_{j}$;

(iii) if $x_{0}, x_{1} \ldots \in X$ then there is an infinite $A \subseteq \mathbb{N}$ such that $x_{i} \leq x_{j}$ for all $i<j$ in $A$ (every infinite sequence has an infinite non-decreasing subsequence);

(iv) for any $S \subseteq X$, there is a finite $T \subseteq S$ such that $\forall x \in S, \exists y \in T$ such that $y \leq x$ (every non-empty subset contains only finitely many non-equivalent minimal elements, and every element lies above at least one such minimal element).

A basic example of a wqo is $(\mathbb{N}, \leq)$, the natural numbers under the usual ordering. In contrast, $(\mathbb{Z}, \leq)$ is not a wqo because it is not well-founded, and $(\mathbb{N}, \mid)$, the natural numbers ordered by divisibility, is not a wqo since the prime numbers form an infinite antichain. Another example of a wqo is $\left(\mathbb{N}^{k}, \leq\right)$, the set of $k$-tuples of natural numbers with component-wise ordering; the result which asserts that this is a wqo is known as Dickson's Lemma. More generally:

Theorem 1.2 The class of well quasi-ordered sets is closed under: (i) taking of subsets; (ii) homomorphic images; (iii) finite unions; (iv) finite cartesian products. 
Given an arbitrary finite alphabet $A$ (with at least two elements), the set of words $A^{*}$ over $A$ is not a wqo under either the lexicographic order nor the factor order (witnessed in both cases by the sequence $a b^{i} a, i=1,2, \ldots$ ). A fundamental result due to G. Higman asserts that $A^{*}$ is a wqo under the subword (subsequence) ordering.

In fact, Higman proves a vastly more general result, which he couches in terms of abstract algebras, i.e. structures of the form $(X, F)$ where $X$ is a set and $F$ is a set of operations on $X$. Both sets are arbitrary and may be infinite. The operations in $F$ are assumed to be finitary (i.e. take a finite number of arguments), and their arities are assumed to be bounded. In other words, if $F_{r}$ denotes the set of all basic operations that take $r$ arguments, then all but finitely many $F_{r}$ are empty. We say that the algebra is minimal if it has no proper subalgebras (subsets of $X$ closed under all operations). Suppose that we have a quasi-order $\leq$ on $X$. This makes $(X, F)$ into an ordered algebra if all operations from $F$ are compatible with $\leq$, i.e. if for all $f \in F_{r}$, and all $x_{1}, \ldots, x_{i-1}, x_{i}^{\prime}, x_{i}^{\prime \prime}, x_{i+1}, \ldots, x_{r} \in X$ with $x_{i}^{\prime} \leq x_{i}^{\prime \prime}$ we have

$$
f\left(x_{1}, \ldots, x_{i-1}, x_{i}^{\prime}, x_{i+1}, \ldots, x_{r}\right) \leq f\left(x_{1}, \ldots, x_{i-1}, x_{i}^{\prime \prime}, x_{i+1}, \ldots, x_{r}\right) .
$$

We say that $\leq$ is a divisibility order if

$$
x_{i} \leq f\left(x_{1}, \ldots, x_{i}, \ldots, x_{r}\right)
$$

for all $f \in F_{r}$ and all $x_{1}, \ldots, x_{r} \in X$. Finally, suppose that each set $F_{r}$ is quasi-ordered in its own right. We say that these quasi-orders are compatible with the quasi-order on $X$ if

$$
f \leq g \Rightarrow f\left(x_{1}, \ldots, x_{r}\right) \leq g\left(x_{1}, \ldots, x_{r}\right)
$$

for all $f, g \in F_{r}$ and all $x_{1}, \ldots, x_{r} \in X$.

Theorem 1.3 (Higman [30, Theorem 1.1]) Any minimal algebra $(X, F)$ with a divisibility order $\leq$, such that all $F_{r}$ are well quasi-ordered and compatible with $\leq$, is itself well quasi-ordered.

It is fair to say that almost every non-trivial proof of well quasiorderedness in mathematics utilises, in one way or another, Higman's Theorem (or its sister, Kruskal's Tree Theorem; see discussion in Subsection 4.2). The theorem is seldom applied in its full generality though, but rather in one of a series of specialisations. Firstly, one can assume that the algebra $(X, F)$ comes equipped with a generating set $A$. By treating the elements of $A$ as nullary operations (constants) we ensure that the algebra is minimal. If in addition we assume that there are only finitely many 
operations of other arities (thus dispensing with the wqo requirement on $F_{r}$ ), we obtain:

Corollary 1.4 Suppose that $(X, F)$ is an algebra, ordered by divisibility, generated by a well quasi-ordered set $A$, and with the set of operations $F$ finite. Then $(X, F)$ is well quasi-ordered.

Obviously, every finite set is well quasi-ordered, so we obtain a further specialisation:

Corollary 1.5 Every finitely generated algebra $(X, F)$, ordered by divisibility and with $F$ finite, is well quasi-ordered.

By specialising Corollary 1.4 in a different direction to the free monoid $A^{*}$, consisting of all the words over alphabet $A$ under the operation of concatenation, which clearly is generated by $A$ itself, we obtain two further corollaries:

Corollary 1.6 If $A$ is a well quasi-ordered alphabet, then the free monoid $A^{*}$ is well quasi-ordered by the domination ordering:

$a_{1} \ldots a_{m} \leq b_{1} \ldots b_{n} \Leftrightarrow\left(\exists 1 \leq j_{1}<\ldots<j_{m} \leq n\right)(\forall i=1, \ldots, m)\left(a_{i} \leq b_{j_{i}}\right)$.

Corollary 1.7 If $A$ is a finite alphabet, then the free monoid $A^{*}$ is well quasi-ordered under the subword ordering:

$a_{1} \ldots a_{m} \leq b_{1} \ldots b_{n} \Leftrightarrow\left(\exists 1 \leq j_{1}<\ldots<j_{m} \leq n\right)(\forall i=1, \ldots, m)\left(a_{i}=b_{j_{i}}\right)$.

Well quasi-order usually makes an appearance when one is interested in certain downward closed classes of mathematical objects. The appropriate order theoretic notions capturing this are ideals, and their duals, filters. Suppose we have quasi-order $(X, \leq)$. A subset $I$ of $X$ is called an ideal or downward closed set if $y \leq x \in I$ implies $y \in I$. Dually, a subset $F$ of $X$ is called a filter if it is upward closed; if $y \leq x$ and $y \in F$ implies $x \in F$. It is clear that the complement of an ideal is a filter, and vice versa. An equivalent condition for $X$ to be a wqo, is that for every filter $F$ of $X$, there exists a finite set $B$ such that $F=\{x \in X:(\exists b \in B)(b \leq x)\}$ (part (iv) of Theorem 1.1). Here $B$ is said to generate $F$; Higman refers to this as the finite basis property. We will more often use the alternative formulation:

Lemma 1.8 A quasi-order $(X, \leq)$ is a wqo precisely if, for every ideal I of $X$, there is a finite set $B$ of forbidden elements such that

$$
I=\operatorname{Av}(B)=\{x \in X:(\forall b \in B)(b \not \leq x)\} .
$$

A minimal such $B$ is often called a basis. 


\section{WQO in combinatorics}

Although well quasi-order originates in the area of order theory, it appears with particular frequency in combinatorics, and indeed seems to be the appropriate language in which to describe and explore various combinatorial situations. We may naturally wonder why this is so. We have seen in the introduction, that if we have a quasi-order $\leq$ on a class $C$ of combinatorial objects, then various helpful consequences follow if $\leq$ happens to be a well quasi-order. If we have a family $X$ in our class $C$ which is downward-closed under our quasi-order, then we know $X$ can be characterized by avoiding a finite number of forbidden objects from our class. As an immediate implication we know that there are only countably many such downward-closed sets (provided that $C$ itself is countable, which it invariably is in classical combinatorial settings), and thus one at least in principle can hope to explicitly characterise (list) them all. By way of contrast, if the class $C$ is not wqo by virtue of an infinite antichain $A$, then by noting that every subset of an antichain is also an antichain, we conclude that there are at least continuum many downward closed classes in this case. Thus well quasi-orderedness can be viewed as a demarcation between 'tame' and 'wild' classes of combinatorial objects.

Some typical specific contexts in which wqo has been investigated in combinatorics are:

- words over an alphabet under the subword ordering;

- graphs under the subgraph ordering;

- graphs under the induced subgraph ordering;

- tournaments under the sub-tournament (which coincides with the induced sub-tournament) ordering;

- permutations under (sub)permutation involvement;

- graphs under the minor ordering;

- trees under homeomorphic embedding.

In fact these diverse contexts can be brought under the same umbrella by considering combinatorial structures as (finite) relational structures, i.e. sets with relations defined on them. Thus, for example, in this language an (undirected) graph is a set with a symmetric binary relation. The model can be further refined by requiring the relation to be irreflexive or reflexive. A permutation can be viewed as a set with two linear orders. Similar descriptions can be given for nearly all common combinatorial 
structures. We will not list all these descriptions here, but refer the reader to [34] for a fairly comprehensive treatment.

In this set-up, all the above orders on combinatorial structures are expressible in terms of homomorphisms. Suppose we have two relational structures $\mathcal{S}=\left(S, R_{i}^{\mathcal{S}}(i \in I)\right)$ and $\mathcal{T}=\left(T, R_{i}^{\mathcal{T}}(i \in I)\right)$ in the same signature (so that the arities of $R_{i}^{\mathcal{S}}$ and $R_{i}^{\mathcal{T}}$ are the same for every $i \in I$ ), and let $\phi: S \rightarrow T$ be a mapping. We say that $\phi$ is:

(i) a homomorphism if

$$
\left(s_{1}, \ldots, s_{k}\right) \in R_{i}^{\mathcal{S}} \Rightarrow\left(\phi\left(s_{1}\right), \ldots, \phi\left(s_{k}\right)\right) \in R_{i}^{\mathcal{T}},
$$

i.e. if $\left.\phi\left(R_{i}^{\mathcal{S}}\right) \subseteq R_{i}^{\mathcal{T}}\right|_{\phi(S)}$;

(ii) a strong homomorphism if $\phi$ is a homomorphism and satisfies $\phi\left(R_{i}^{\mathcal{S}}\right)=$ $\left.R_{i}^{\mathcal{T}}\right|_{\phi(S)}$

(iii) an M-strong homomorphism if $\phi$ is a homomorphism such that

$$
\left(s_{1}, \ldots, s_{k}\right) \in R_{i}^{\mathcal{S}} \Leftrightarrow\left(\phi\left(s_{1}\right), \ldots, \phi\left(s_{k}\right)\right) \in R_{i}^{\mathcal{T}},
$$

i.e. $\phi\left(R_{i}^{\mathcal{S}}\right)=\left.R_{i}^{\mathcal{T}}\right|_{\phi(S)}$ and $\phi\left(\overline{R_{i}^{\mathcal{S}}}\right)=\left.\overline{R_{i}^{\mathcal{T}}}\right|_{\phi(S)}$, where bars denote complementation.

The ' $\mathrm{M}$ ' in the final definition refers to the model theoretic definition of a strong embedding (see, for example, [31, Chapter 1]). A homomorphism is an embedding if it is injective, and is an epimorphism if it is onto. Note that an embedding $\phi: S \rightarrow T$ is M-strong if and only if it is strong.

Now, the subgraph ordering $G \leq H$ on the class of graphs becomes simply the existence of an embedding $G \rightarrow H$, while the induced subgraph ordering means the existence of such an embedding that is required to be strong. For tournaments, embeddings and strong embeddings coincide, and so the subtournament and induced subtournament orderings are identical. Slightly less obviously, the subword ordering on words over an alphabet $A$ can be interpreted as the existence of an embedding (equivalently, strong embedding), when words are appropriately represented as relational structures. This can be done by viewing a word $w$ as a set $X$ of size $|w|$ (representing the letters of $w$ ), with a linear ordering defined on it (specifying the order of letters in $w$ ), and a family of unary relations $f_{a}$ ( $a \in A$ ) such that for every $x \in X$ precisely one $f_{a}(x)$ is true (thus specifying to which letter of $A$ an element $x$ corresponds). For more details we refer the reader to [34, Subsection 2.2]. Permutation involvement is again the same as existence of (strong) embeddings.

Even less obviously, the graph minor relation can be interpreted in terms of homomorphisms. To do this, we need to consider graphs in their 
reflexive representation (otherwise a homomorphism could not contract an edge). A graph $G$ is a minor of a graph $H$ if there exists a graph $K$, an embedding $K \rightarrow H$, and an epimorphism $K \rightarrow G$ the kernel classes (or fibres) of which are connected.

In this paper we restrict our attention to finite combinatorial structures, with a consequence that all the homomorphism-defined orders are in fact partial orders, because they respect size. The single exception is the homomorphism order; see Section 6. However, if one extends one's field of study to infinite structures, then they all become genuine quasi-orders. For instance, it is perfectly possible for two non-isomorphic graphs $G$ and $H$ to mutually embed into each other. Generally, we follow the prevailing usage in literature, and favour the term quasi-order in preference to partial order.

Returning to our survey of wqo in combinatorics, we note that the presence of this property offers various algorithmic advantages. Probably the best example of this is membership testing for downward closed subclasses of a class $C$ that is wqo under taking of substructures or induced substructures. Indeed, each such subclass $X$ is given by its finite basis $\left\{B_{1}, \ldots, B_{k}\right\}$. To test whether an object $A$ belongs to $X$ we need to check whether it avoids each $B_{i}$. This can be done by examining all subsets of $A$ having size $\left|B_{i}\right|$, a process that is polynomial in the size of $A$. So we see that membership in downward closed subclasses of $C$ is of polynomial complexity.

Perusing the literature, we see that historically many combinatorial structures have been approached via the question of whether some of the objects in a given set are 'involved' in the others, which very naturally leads to the topic of wqo. For example, Vazsonyi's conjecture, made in the 1940s, hypothesises that any infinite collection of finite trees must contain two trees such that one is homeomorphically embeddable in the other (topological minor relation); this can equivalently be viewed as conjecturing that the quasi-order of trees under the topological minor relation is wqo (see [42]).

Some natural membership questions, posed early in the study of graph theory, turned out to have answers expressible in terms of whether a graph avoids certain forbidden graphs, also naturally leading to consideration of wqo. For example, in 1930, Kuratowski ([43]) showed that being planar means containing no subdivision of the complete graph $K_{5}$ or the complete bipartite graph $K_{3,3}$, while in 1937, Wagner [63] proved that being planar is equivalent to avoiding these two graphs as minors. We may observe that the property of being planar is closed under the taking of minors. This leads naturally to the more general question of whether, for a property $P$ of graphs closed under minors, there exists a finite set of graphs such 
that a graph possesses property $P$ if and only if it avoids all the graphs in the set as minors? (A result of Robertson and Seymour tells us that the class of graphs under minor order form a wqo, and hence every ideal does indeed have a finite set of forbidden elements.) Another example is that the property of being a cograph (a graph that can be generated from the single-vertex graph by the taking of complements and disjoint union) turns out to be equivalent to being $P_{4}$ induced-subgraph-free ([15]).

A possible way to view wqo investigations in combinatorics is as follows. One is originally and naturally interested in combinatorial structures and their substructures (induced or not). Unfortunately, with the exception of words over a finite alphabet, the resulting ordering will not be wqo for any other full class of combinatorial objects (e.g. all graphs, or all permutations). Still, wqo is a desirable property, and one wants to move in its direction. One way of doing it is by 'freeing up' the ordering, such as in the graph minor or homeomorphic embedding contexts. Another way is to try and identify the subclasses of $C$ which are wqo, even if $C$ itself is not.

Another aspect of wqo which can offer new insights and useful methods for combinatorial problems, but as yet has been surprisingly little-studied, is its connection with regular languages and rational generating functions. A natural illustration of this occurs in the setting of words over a finite alphabet. We may naturally ask, whether something similar can be fruitfully carried out in classes of other combinatorial objects.

In the next three sections we will present three case studies, in the form of brief surveys of wqo considerations for words, graphs and permutations. We have chosen these three not only because they comprise much of the existing work on wqo but also because they exhibit different combinations of the viewpoints outlined above. The results for words are classical and can serve as an exemplar and a tool for other, more complicated contexts. In graph theory we have the largest body of results demarcating the boundary between wqo and non-wqo classes. Finally in permutations we encounter the most active attempts at interweaving wqo with structural and enumerative considerations. In Section 6 as an attempt to bring these strands and areas of combinatorics closer together, we will propose a closer and more systematic study of homomorphism orderings.

\section{Case study 1: words}

Let $A$ be a finite alphabet, and let $A^{*}$ be the set of all words (i.e. sequences of symbols) over $A$. Recall that the subword ordering on $A^{*}$ is defined by $u \leq v$ if $u$ is a subword (i.e. subsequence) of $v$. Let $X$ be an order ideal in this ordering, i.e. a non-empty set such that $u \leq v \in X$ 
implies $u \in X$. The complement $A^{*} \backslash X$ is a filter, i.e. it is upward closed. By Higman's Theorem (Corollary 1.7) the set $A^{*}$ is wqo, and so $A^{*} \backslash X$ has finitely many minimal elements $B=\left\{w^{(i)}=a_{1}^{(i)} \ldots a_{m_{i}}^{(i)}: i=1, \ldots, n\right\}$. Furthermore, the set $X$ is the avoidance set of $B$, i.e.

$$
X=\operatorname{Av}(B)=\left\{u \in A^{*}: w^{(i)} \not \leq u \text { for } i=1, \ldots, n\right\} .
$$

Now, the set of all words containing a subword $w=a_{1} \ldots a_{m}$ can be expressed as $A^{*} a_{1} A^{*} a_{2} \ldots A^{*} a_{m} A^{*}$. Hence

$$
X=A^{*} \backslash \bigcup_{i=1}^{n} A^{*} a_{1}^{(i)} A^{*} a_{2}^{(i)} \ldots A^{*} a_{m_{i}}^{(i)} A^{*} .
$$

This is a (very simple) example of a regular expression from formal language theory, proving that $X$ is a regular language. By Kleene's Theorem every regular language is accepted by a finite state automaton. It is in turn known that the enumeration sequence of such a language has a rational generating function. This is actually easy to see. Recall that a (deterministic) finite state automaton consists of a finite set of vertices $Q$, a finite input alphabet $A$, and a transition function $\tau: Q \times A \rightarrow Q$. One vertex $q_{I} \in Q$ is designated as a start state, and there is a set of final states $Q_{F} \subseteq Q$. A relationship $\tau\left(q_{1}, a\right)=q_{2}$ is interpreted as a directed edge from $q_{1}$ to $q_{2}$ labelled by $a$. A word $w=a_{1} \ldots a_{m} \in A^{*}$ is accepted by the automaton if starting at $q_{I}$ and successively following the edges labelled $a_{1}, \ldots, a_{m}$ ends in a final state. The set $L$ of all the accepted words is the language accepted by the automaton. Now, for $q \in Q$, let $L_{q}$ be the set of words $w$ such that reading of $w$ from $q_{I}$ terminates in $q$, and let $f_{q}$ be the generating function for $L_{q}$. Note that a word $w=w^{\prime} a$ is in $L_{q}$ if and only if $w^{\prime} \in L_{q^{\prime}}$ and $\tau\left(q^{\prime}, a\right)=q$. Furthermore, note that $f_{q}(0)=1$ if and only if $q=q_{I}$, and $f_{q}(0)=0$ otherwise. This yields the equations

$$
\begin{aligned}
& f_{q_{I}}=1+x \sum_{\tau\left(q^{\prime}, a\right)=q_{I}} f_{q^{\prime}}, \\
& f_{q}=x \sum_{\tau\left(q^{\prime}, a\right)=q} f_{q^{\prime}} \quad\left(\text { when } q \neq q_{I}\right) .
\end{aligned}
$$

These equations are clearly linear in the $f_{q}$, and the coefficients are polynomial (indeed, linear) in $x$. Thus, solving this system yields each $f_{q}$ as a rational function in $x$, and the generating function for the entire language is $f=\sum_{q \in Q_{F}} f_{q}$.

All the above material is folklore, and can be found in standard combinatorics textbooks (see for instance Section 8.1 of [22] or Section 6.5 of [61]) 
expressed in different degrees of technical sophistication. By presenting it briefly here, in an elementary form, we hope offers the reader a blueprint as to how words, wqo, and Higman's Theorem should combine together to yield structural and enumerative results. We also mention in closing that this has a number of computational and computability consequences. For instance, the above account represents a constructive method for obtaining the generating function from the automaton, or indeed from a finite set of forbidden subwords. Also, the membership problem for a regular language is decidable in linear time (passing the word through the automaton), and a whole other host of properties is decidable; see for instance [32, Section 3.3]. An excellent overview of the uses of formal language theory in combinatorics can be found in [9].

\section{Case study 2: graphs}

In this section, we consider the graph theoretic context. We mainly have in mind 'standard' graphs, but also discuss digraphs, and special classes such as trees and tournaments. The study of graphs and wqo has a long history, arguably originating with the work of Kuratowski [43] and Wagner [63] in the 1930s, with early key results by Kruskal [41] and NashWilliams [53] in the 1960s. There is now a wealth of results, covering many different varieties of graphs and possible orderings. Very generally speaking, one is hoping to establish wqo, but the original 'big' class (e.g. all graphs) under the original 'natural' order (e.g. subgraphs) is quite far from possessing this property. This then leads to investigating subclasses, or variations, or different possible orders. As a result, the great majority of the results in the literature are asserting wqo or its absence in one of the contexts arising in this way.

We begin by giving some definitions of graph operations and hence graph relations.

Definition 4.1 Let $G=(V, E)$ be a graph. We define the following operations on $G$ :

- Removing a vertex $v$ : removing $v$ from the vertex set $V$, and removing all edges incident with $v$ from the edge set $E$.

- Removing an edge e: removing $e$ from the edge set $E$.

- Suppressing a vertex $v$ of degree two: removing $v$ from the vertex set $V$ and replacing the two edges incident to $v$ by a single edge. 
- Contracting an edge $e=u v$ : removing $u$ and $v$ from $V$, and introducing a new vertex $z$ and edges such that $z$ is adjacent to all vertices which were adjacent to $u$ or $v$.

Now, we may define the following orderings. We present them in a way which shows how each definition increasingly 'frees-up' the orderings ([26]), as described above.

Definition 4.2 Let $H, G$ be graphs.

- $H$ is an induced subgraph of $G$ if $H$ can be obtained from $G$ by a sequence of vertex removals.

- $H$ is a subgraph of $G$ if $H$ can be obtained from $G$ by a sequence of vertex and edge removals.

- $H$ is a topological minor of $G$ if $H$ can be obtained from $G$ by a sequence of vertex removals, edge removals, and suppressions of vertices of degree two.

- $H$ is a minor of $G$ if $H$ can be obtained from $G$ by a sequence of vertex removals, edge removals, and edge contractions.

Observe that suppression of a vertex of degree two can be viewed as the contraction of either of the two incident edges, so that this is indeed a hierarchy: $H$ an induced subgraph of $G$, implies $H$ is a subgraph of $G$, which in turn implies that $H$ is a topological minor of $G$, and hence that $H$ is a minor of $G$. It can be shown that all of these graph relations form quasi-orders on the set of finite graphs. We will see in the ensuing subsections that, initially far from being wqo, they move progressively towards possessing this property. We will also discuss a related ordering by immersions.

\subsection{Subgraph order}

The class of all graphs is not a wqo under either the subgraph or induced subgraph order; in both cases, an infinite antichain is provided by the set of cycles $C_{k}$ or the set of double-ended-forks $F_{k}$; see Figure 1 . The latter antichain also witnesses that the class of all trees is not a wqo under subgraph or induced subgraph order.

It is, however, known that the graphs avoiding the path $P_{k}$ of length $k$ are wqo by the subgraph relation for any value of $k([19])$, and that $P_{4}$-free graphs are wqo by the induced subgraph relation [17]. Indeed $P_{4}$ is the unique maximal graph $G$ such that the class of all $G$-free graphs is 


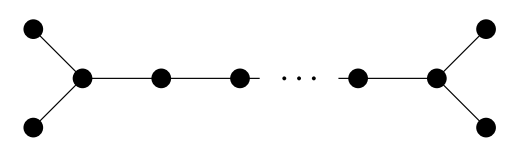

Figure 1: A typical double-ended fork $F_{k}$

wqo under induced subgraph order; in other words, if the class of $G$-free graphs is wqo then $G$ is necessarily an induced subgraph of $P_{4}$ ([17]). Special classes, such as the set of bipartite graphs, have also been investigated under induced subgraph order. For example, it is conjectured in [19] that the $P_{7}$-free bipartite graphs are not wqo under the induced subgraph relation; in [36] it is shown that this is indeed the case, but that the $P_{6}$-free bipartite graphs are wqo. In [37], wqo classes of graphs defined by more than one induced subgraph obstruction are considered.

In [19], the wqo ideals in the class of graphs under subgraph and induced subgraph order were characterized in terms of forbidden subgraphs.

Theorem 4.3 (Ding, [19]) Let $\mathcal{F}$ be an ideal of graphs with respect to subgraph relation. Then the following are equivalent:

- $\mathcal{F}$ is a wqo under the subgraph ordering;

- $\mathcal{F}$ is a wqo under the induced subgraph ordering;

- $\mathcal{F}$ contains only finitely many graphs $C_{n}$ (cycles) and $F_{n}$ (doubleended forks).

One possible interpretation of this result is that it is algorithmically decidable whether an ideal of graphs defined by finitely many obstructions is wqo or not. Indeed suppose that we have such an ideal $C=$ $\operatorname{Av}\left(G_{1}, \ldots, G_{k}\right)$. According to Ding's Theorem, $C$ will be wqo if and only if for some $n_{0}$ we have $C_{n}, F_{n} \notin C$ for all $n \geq n_{0}$. This in turn is equivalent to each $C_{n}$ and each $F_{n}\left(n \geq n_{0}\right)$ containing some $G_{i}$. Now, observe that for a graph $G$ with $m$ vertices we have $G \leq C_{p}$ if and only if $G \leq C_{q}$ for all $p, q>m$. An analogous assertion holds for the $F_{p}$. It follows that $C$ is wqo if and only if $C_{n_{1}}, F_{n_{1}} \notin C$, where $n_{1}=\max \left(\left|G_{1}\right|, \ldots,\left|G_{k}\right|\right)+5$. This property is clearly algorithmically decidable, by listing all the subgraphs of $C_{n_{1}}$ and of $F_{n_{1}}$ and checking they both intersect $\left\{G_{1}, \ldots, G_{k}\right\}$. It is worth noting that no such algorithm is known for the induced subgraph ordering. Recent related work, which also reflects the position of trees 
somehow being the key for the wqo/non-wqo distinction for graphs, is the result that graphs of finite tree-depth ordered by the induced subgraph relation form a well quasi-order ([55, Lemma 6.13]).

In the absence of a characterisation of wqo ideals of graphs under the induced subgraph ordering, one can attempt such a generalisation in the presence of additional restrictions. One such approach is to consider labelled graphs (or, equivalently, vertex coloured graphs). A collection of graphs $C$ is said to be $n$-wqo (by the induced subgraph order) if the set of all $n$-labellings of members of $C$ is wqo by the induced (labelled) subgraph relation. Clearly, in this terminology, the property of being wqo is equivalent to being 1-wqo. On the other hand, the set of paths $\left\{P_{n}\right\}$ is wqo but not 2 -wqo. Rather curiously, every induced subgraph ideal of graphs which is 2-wqo is finitely based [57]. It has been conjectured (by Pouzet [57] in 1972, and Kř́̌z and Thomas [40] in 1990) that for an ideal in the induced subgraph order, being 2 -wqo is equivalent to being $n$-wqo for all $n$. For more on this, and a possible proof strategy for the conjecture, see [16]. This conjecture is not true in a more general setting of labelled categories, as demonstrated by Kř́ǐ and Sgall [39].

Since we do not have a wqo for graphs, we cannot have one for digraphs. We can try restricting our attention to special types of digraphs, for example tournaments, but in fact the subgraph relations do not give rise to a wqo for the class of all tournaments $([29],[45])$. The specialization to classes of tournaments avoiding a given tournament as a subgraph were considered by Cherlin and Latka (see [13] for a summary of results in this area):

\section{Theorem 4.4}

- Let $L$ be a finite linear tournament (i.e. isomorphic to the structure $(\{1, \ldots, n\},<)$ for some $n)$. Then the $L$-free tournaments are wqo (indeed of bounded size).

- If $L$ is a non-linear tournament, with at least 7 vertices, then the L-free tournaments are not wqo (two antichains witness this in all cases).

The above theorem is sufficient to conclude that it is algorithmically decidable whether a class of tournaments avoiding a single obstruction is wqo, as it gives a characterisation of wqo for all but finitely many tournaments $L$ (the exceptions being non-linear tournaments of size less than 7). These exceptions have in fact also been analysed; see the discussion in [13, Subsection 2.2]. A question arises whether wqo is decidable for arbitrary ideals 


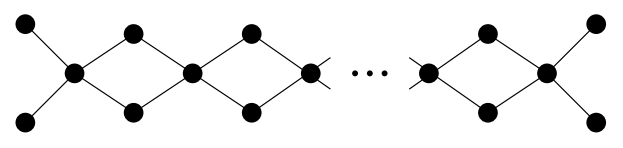

Figure 2: A modified double-ended fork

of tournaments under the subgraph ordering defined by finitely many obstructions. This is at present an open question. We note that work on wqo questions in tournaments was motivated by the analysis of homogeneous directed graphs ([12]). It was shown by Henson in $1972([29])$ that any antichain of tournaments translates into uncountably many homogeneous digraphs, and in fact this accounts for all but countably many of these digraphs. Various questions about the digraphs can then be mapped back into questions about the structure of the quasi-order of finite tournaments.

The above summary suggests that, if we are hoping to obtain wqo results for the class of all graphs, or classes such as the class of trees or of tournaments, it might be fruitful to consider other orders.

\subsection{Topological minor}

The topological minor, also called the homeomorphic embedding relation, has been much-studied historically, and was the setting for some of the earliest results in this area. We defined the topological minor in terms of suppressions of vertices of degree two. An alternative expression is that a graph $H$ is a topological minor of a graph $G$ if a subdivision of $H$ is isomorphic to a subgraph of $G$, where a subdivision means replacement of edges by paths.

The class of graphs is not wqo by the topological minor ordering. Several antichains witnesses this. One such is $F_{k}^{\prime}$, which can be constructed from the double ended forks $F_{k}$ by doubling every edge in the central part of the fork (see [48]). This of course is an example in the class of graphs with multiple edges. A suitable modification for the class of simple graphs is shown in Figure 2.

However, this quasi-order is not very far away from being wqo in some sense. For instance, Mader [49] proves that the class of all graphs that do not have $k$ disjoint cycles as topological minors for some $k \in \mathbb{N}$ are wqo.

In a celebrated early result Kruskal [41] proves:

Theorem 4.5 The class of all trees is wqo under the topological minor 
ordering.

This effectively establishes trees as, in a way, being at the boundary between wqo and non-wqo in the world of graphs, a frequently occurring theme in subsequent developments. This could perhaps be elucidated by noticing that words could be interpreted as (labelled) paths, and that topological minor ordering would coincide with the subword ordering in this interpretation. General finite trees could then be viewed as finite sets of overlapping words.

An improved proof of Kruskal's tree theorem was given in 1963 by Nash-Williams [53]. Nash-Williams [54] went on to prove the analogous result for the class of all trees (finite or infinite). In fact all these results are proved in greater generality for trees labelled by elements from a wqo set. Nash-Williams also introduces a useful strengthening of wqo which he terms a better quasi-ordering. It is in this form that Kruskal's tree theorem is used as a key ingredient in the proof of the graph minor theorem (see below).

It is also worth noting that, as Kruskal himself points out, his tree theorem contains Higman's Theorem 1.4 as a special case. This can be intuitively understood by recalling that the free or term algebra over a generating set $X$ consists of all formal expressions that can be built from basic operations $F$ and elements of $X$ treated as letters. Then it is easy to see that all elements in such an algebra can be represented as trees, with a vertex representing an operation (and is labelled by that operation), and its children are the arguments. It is an exercise to translate Higman's conditions into the topological minor ordering, and derive Higman's Theorem. The final step is provided by the observation that every algebra of type $F$ is a homomorphic image of the term algebra, and that wqo is preserved under homomorphisms.

Returning to the class of all graphs, it transpires that the antichain $F_{k}^{\prime}$ exhibited above is in a sense the only one. Let $P_{k}^{\prime}$ be the central doubled path in $F_{k}^{\prime}$, or, equivalently, the path $P_{k}$ with every edge doubled. Clearly, if an ideal $I$ contains infinitely many $F_{k}^{\prime}$ (and hence is not wqo) then it also contains all $P_{k}^{\prime}$. On the other hand, we have:

Theorem 4.6 The class of graphs avoiding some $P_{k}^{\prime}$ as a topological minor is wqo.

This was originally conjectured by Robertson in 1980s (unpublished), and only recently proved by Liu [48]. Note that this is very close to a (constructive) characterisation of wqo ideals under the topological minor ordering. In fact Ding [20], who proved Robertson's Conjecture in the 
special case of minor (as opposed to topological minor) closed classes, provides such a characterisation in this case: a minor closed class is wqo by the topological minor order if and only if it avoids some graph $B_{k}^{\prime}$, obtained from $P_{k}^{\prime}$ by attaching two loops at each of the two ends.

\subsection{Minor order}

Now we reach the minor order, which is the setting for perhaps the bestknown wqo result on graphs. There are various alternative expressions of the definition: for example, a graph $G$ is a minor of a graph $H$ if $G$ can be obtained from a subgraph of $H$ by successively contracting edges, or by collapsing connected subgraphs, see [18, Section 1.7].

A result from the 1930s (often called Kuratowski's Theorem, but in fact due to Wagner in its usually-quoted form) states that a finite graph is planar precisely if it avoids $K_{5}$ and $K_{3,3}$ as minors. After a lengthy proof process (in a series of papers spanning 21 years from 1983 to 2004), Robertson and Seymour proved in [59] that:

Theorem 4.7 The class of graphs is a wqo under minor order.

The definition of minor can be extended to digraphs in various ways (for digraphs, unlike graphs, contracting edges or connected subgraphs yields different concepts). If we define a digraph minor by saying a digraph $H$ is a minor of a digraph $G$ if $H$ can be obtained from a subdigraph of $G$ by repeatedly contracting a strongly-connected subdigraph to a vertex, we can consider the quasi-order of digraphs under the directed minor relation. This is not a wqo - for example, the directed cycles form an infinite antichain. However, we can consider subclasses; for example, it can be shown $([35])$ that

Theorem 4.8 (Kim [35]) The class of all finite tournaments is a wqo under minor order.

\subsection{Immersion order}

Finally, we mention one other minor-type order which has received attention and yielded positive wqo results - the immersion order (originally defined by Nash-Williams). Graph $H$ is said to be an immersion minor of graph $G$ is there is an injective mapping from $V(H)$ to $V(G)$ such that the images of adjacent elements of $H$ are connected in $G$ by edgedisjoint paths. An alternative definition via graph operations is in terms of lifting: a pair of adjacent edges $u v$ and $v w$ with $u \neq v \neq w$ is lifted by deleting the edges $u v$ and $v w$ and adding the edge $u w$. A graph $H$ is 
said to be immersed in a graph $G$ precisely if a graph isomorphic to $H$ can be obtained from $G$ by lifting pairs of edges and taking a subgraph [8]. There are also the weak and strong immersion orders (the weak version also allows the operation of vertex-splitting). It was shown in [60] that

Theorem 4.9 The class of graphs is a wqo under weak immersion order.

Various consequences of this, in terms of finite bases, are explored in [27]; in particular it is hoped to lead to faster membership algorithms for the general immersion case. It has been conjectured by Seymour that the class of graphs is also a wqo under strong immersion order.

The definition of immersion can be extended to digraphs, by replacing paths with directed paths. It is not the case that the class of all digraphs is a wqo under weak immersion [35], but the class of tournaments is wqo under immersion, and it was shown that in fact the class of tournaments is wqo under strong immersion [14].

\section{Case study 3: permutations}

Compared to the study of graphs, investigation of wqo in permutations is much younger, with the first results appearing in the early 2000s; see [7]. Furthermore, permutations themselves are fairly restricted combinatorial structures, with no obvious variations available, and with essentially all homomorphism-related orders reducing to the subpermutation ordering; see [34]. Thus the overall body of results is considerably smaller than in the case of graphs. And yet, these restrictions have acted as a catalyst for directing research towards slightly different types of questions. One outcome of this is that interesting links with words and Higman's Theorem have emerged, leading to structural and enumerative consequences seemingly quite remote from the wqo itself. Here we review some developments of this nature.

For two permutations $\alpha=a_{1} \ldots a_{m}, \beta=b_{1} \ldots b_{n}$, we say that $\alpha$ is involved or contained in $\beta$ (denoted $\alpha \leq \beta$ ) if $\beta$ contains a subsequence $b_{k_{1}} \ldots b_{k_{m}}$ order isomorphic to $\alpha$, i.e. satisfying $a_{i}<a_{j}$ if and only if $b_{k_{i}}<b_{k_{j}}$. If permutations are regarded as relational structures with two linear orders, then this definition coincides with the notion of (induced) substructure. Permutations can also be viewed as (equivalence classes) of sets of $n$ points in a plane (arising from their plots as bijections), in which case involvement becomes simply presence of the corresponding pattern of $\alpha$ in the plot of $\beta$; see Figure 3 for illustration. The ideals of permutations under this order are referred to as pattern (avoidance) classes. 


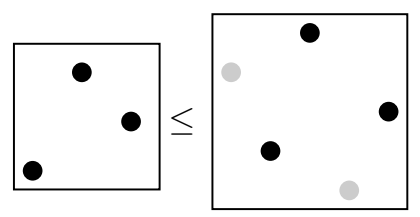

Figure 3: Plot of the permutation 132 and the same permutation as a subpattern of 42513

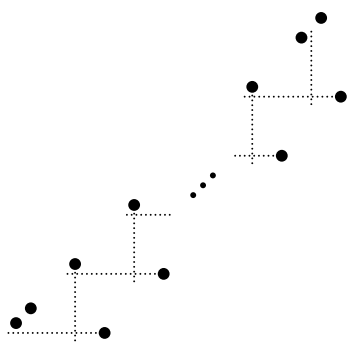

Figure 4: A typical permutation in the antichain $\mathcal{A}$

The poset of all permutations is not wqo, and there are many antichains witnessing this. Perhaps the easiest one is the oscillating antichain $\mathcal{A}=\left\{A_{n}: n \in \mathbb{N}\right\}$, depicted in figure 4. This antichain, and its left-right reflection, suffice to prove that the only singly-based wqo classes are $\operatorname{Av}(12)$ (increases), $\operatorname{Av}(21)$ (decreases) and $\operatorname{Av}(231)$ and its four symmetries. Murphy's thesis [51] contains an extensive 'Bibliothek' of antichains. A common feature of all the antichains presented there is that the main body of each permutation consists of what was later termed a pin sequence [10], with an irregularity at the beginning and the end. This, and some subsequent results on pin sequences [11] give some hope that a (constructive) characterisation of finitely based wqo classes may be possible, but so far this has been elusive. 


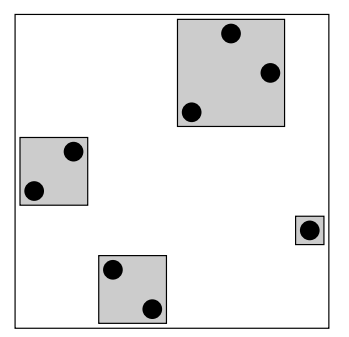

Figure 5: Inflation $3142[12,21,132,1]=45216873$

\subsection{Classes with finitely many simple permutations}

The inflation of a permutation $\alpha=a_{1} \ldots a_{m}$ by permutations $\delta_{1}, \ldots, \delta_{m}$ is the permutation $\alpha\left[\delta_{1}, \ldots, \delta_{m}\right]$ obtained by replacing each point $a_{i}$ of $\alpha$ by a set of contiguous points representing $\delta_{i}$. For an illustration see Figure 5. Observe that the inflation $\alpha\left[\delta_{1}, \ldots, \delta_{m}\right]$ contains all $\delta_{i}$, as well as $\alpha$, as subpermutations. Thus if $X$ is a pattern class and $\alpha\left[\delta_{1}, \ldots, \delta_{m}\right] \in X$, then necessarily $\alpha, \delta_{1}, \ldots, \delta_{m} \in X$. For sets $A$ and $B$ the inflation of $A$ by $B$ is

$$
A[B]=\left\{\alpha\left[\beta_{1}, \ldots, \beta_{m}\right]: \alpha=a_{1} \ldots a_{m} \in A, \beta_{1}, \ldots, \beta_{m} \in B\right\}
$$

When $A$ and $B$ are pattern classes, then so is $A[B]$. The (wreath) closure of a set $X$ is

$$
\langle X\rangle=X \cup X[X] \cup X[X[X]] \cup \ldots
$$

This is the smallest class containing $X$ closed under inflations.

A permutation is said to be simple if it cannot be expressed as an inflation in a non-trivial way. Simple permutations are basic blocks from which all other permutations are built by means of successive inflations. Albert and Atkinson [1] develop a theory of classes with finitely many simple permutations. Let $A$ be such a class and let $S$ be its finite set of simple permutations. Let $W=\langle A\rangle$ be the closure of $A$ and note that $W=\langle S\rangle$. For each $\sigma=a_{1} \ldots a_{m} \in S$ define an $m$-ary operation $f_{\sigma}$ :

$$
f_{\sigma}\left(\alpha_{1}, \ldots, \alpha_{m}\right)=\sigma\left[\alpha_{1}, \ldots, \alpha_{m}\right]
$$

Clearly, $W$ is closed under all these operations, and is generated by the set $S$. Now, as $S$ is finite then we have a finitely generated algebra with finitely many basic operations, and it is easy to see that Higman's Theorem (in the form of Corollary 1.5) applies, and $W$ is wqo. But $A \subseteq W$ and 
so $A$ is wqo as well. Furthermore, because $W$ is defined by a specific construction from a finite set $S$, it is possible to show that $W$ is finitely based, and then it follows that $A$ is finitely based as well. It is relatively easy to exhibit an algebraic generating function for $W$. A more detailed analysis of permutations in $A$, similar in essence to that of words given in Section 3 and likewise dependent on the finiteness of the obstruction set, then yields an algebraic generating function for $A$ itself.

Theorem 5.1 (Albert, Atkinson [1]) Every pattern class containing only finitely many simple permutations is wqo, finitely based and has an algebraic generating function.

For instance, it easily follows that all subclasses of $\operatorname{Av}(231)$ have algebraic generating functions. In fact, with some further analysis, the authors prove that all proper such subclasses have rational generating functions. All the results in this paper are constructive, allowing, at least in principle, generating functions to be computed from the set of simple permutations and the basis. To complement this, Brignall, Ruškuc and Vatter [11] prove that it is decidable whether the set of simple permutations in a pattern class given by a finite basis is finite.

\subsection{Geometric grid classes}

The poset of all permutations under involvement is fairly 'wild', and so is the collection of all pattern classes. Considerable effort has therefore gone into trying to identify some 'tame' classes which can be used as building blocks for more general ones. One such attempt, heavily drawing on the geometric intuition of permutations as point plots in the plane, are the so-called grid classes. Let $M=\left(m_{i j}\right)_{p \times q}$ be a matrix with entries from $\{ \pm 1,0\}$. The grid class $\operatorname{Grid}(M)$ defined by $M$ is the collection of all permutations whose plot can be partitioned into a $p \times q$ grid such that the content of the cell $(i, j)$ is increasing/decreasing/empty if $m_{i j}$ equals $+1 /-1 / 0$; see Figure 6 for an illustration.

Grid classes are a promising structural tool, but at present the analysis of their structure and properties appears to be difficult. It is conjectured that they are all finitely based and have algebraic generating functions, but no proof is known as yet. However, there is a pleasing result characterising when they are wqo. It is couched in terms of a graph $\Gamma(M)$, the $(p, q)$ bipartite graph with adjacency matrix $\left(\left|m_{i j}\right|\right)_{p \times q}$.

Theorem 5.2 (Murphy, Vatter [52]) $\operatorname{Grid}(M)$ is wqo if and only if $\Gamma(M)$ is a forest. 


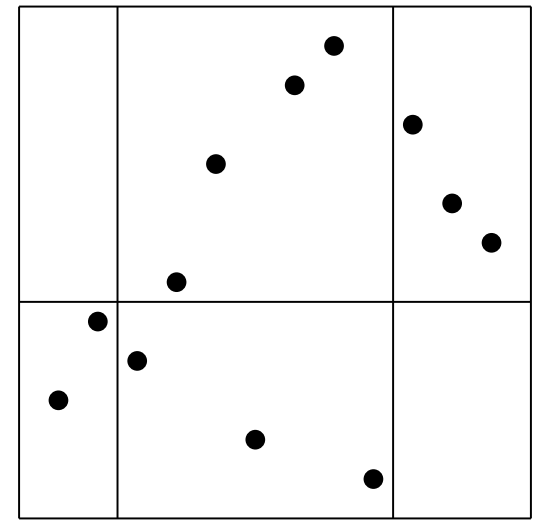

Figure 6 : The permutation $(3,5,4,6,9,2,11,12,1,10,8,7)$ as a member of $\operatorname{Grid}\left(\begin{array}{rrr}0 & 1 & -1 \\ 1 & -1 & 0\end{array}\right)$

The geometric grid class $\operatorname{Geom}(M)$ is an important subclass of $\operatorname{Grid}(M)$, and is obtained as follows. Fix a $p \times q$ rectangular grid in the plane. In the cell $(i, j)$ draw the SW-NE diagonal if $m_{i j}=1$, the NE-SW diagonal if $m_{i j}=-1$, and leave it empty if $m_{i j}=0 . \operatorname{Geom}(M)$ is the set of all permutations that can be plotted on the resulting set of diagonals. This is illustrated in Figure 7.

The remarkable fact about $\operatorname{Geom}(M)$ is that it admits a natural encoding by words over a finite alphabet, which works as follows. First observe that without loss we can assume that all the diagonals can be oriented, so that those belonging to the same row share the same up-down orientation, while those in the same column have the same left-right orientation. If this is not true for the original matrix $M$, it can be 'mended' by enlarging $M$, replacing each each entry $1,-1,0$ by $\left(\begin{array}{ll}0 & 1 \\ 1 & 0\end{array}\right),\left(\begin{array}{ll}1 & 0 \\ 0 & 1\end{array}\right),\left(\begin{array}{ll}0 & 0 \\ 0 & 0\end{array}\right)$, respectively. The alphabet will be $A=\left\{a_{i j}: m_{i j}= \pm 1\right\}$. Given a word $w=a_{i_{1} j_{1}} \ldots a_{i_{n} j_{n}} \in A^{*}$, the permutation $\phi(w)$ is obtained as follows. Pick real numbers $0<d_{1}<d_{2}<\cdots<d_{n}<d$, where $d$ is the common length of all the diagonals. Then for each $k=1, \ldots, n$ place a point on the diagonal in the cell $\left(i_{k}, j_{k}\right)$ distance $d_{k}$ from the base point. This process is illustrated in Figure 8.

The assignment $w \mapsto \phi(w)$ defines a surjective, length-preserving, finite-to-one mapping $A^{*} \rightarrow \operatorname{Geom}(M)$. Furthermore, it is order-preserving, 


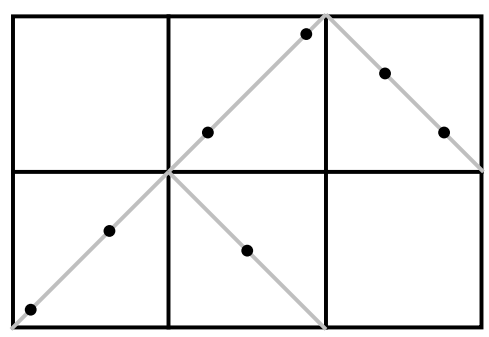

Figure 7: 1352764 as a member of Geom $\left(\begin{array}{rrr}0 & 1 & -1 \\ 1 & -1 & 0\end{array}\right)$

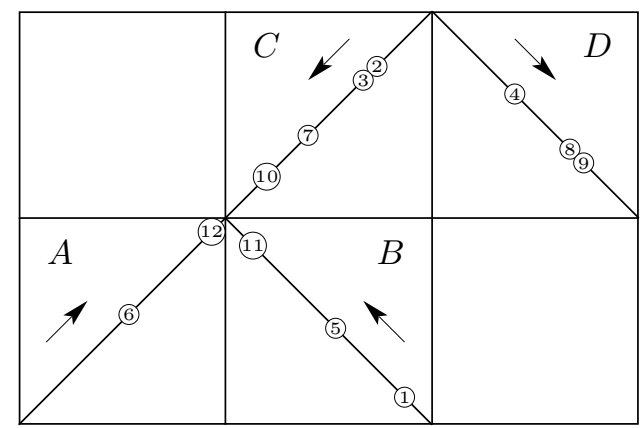

Figure 8: An illustration of the process that yields $\phi(B C C D B A C D D C B A)=(3,5,4,6,9,2,11,12,1,10,8,7)$. The points are numbered in order in which they are inserted. 
in the sense that if $u \leq v$ for $u, v \in A^{*}$ under the subword ordering then $\phi(u) \leq \phi(v)$ under the permutation containment. It immediately follows from Higman's Theorem (in the form of Corollary 1.7) that $\operatorname{Geom}(M)$ is wqo, and a little more work shows it is finitely based as well. By analysing preimages of permutations under $\phi$, we obtain a regular language $L \subseteq A^{*}$ such that $\phi$ maps $L$ bijectively onto $\operatorname{Geom}(M)$. From here it follows that $\operatorname{Geom}(M)$ has a rational generating function. Furthermore, one can look at subclasses of $\operatorname{Geom}(M)$ (termed geometrically griddable classes), use wqo for $\operatorname{Geom}(M)$ to see they are defined by finitely many obstructions, and conclude that they are finite unions of geometric grid classes and possess the above pleasing properties. To summarise:

Theorem 5.3 (Albert et al. [2]) Every geometric grid class Geom $(M)$ is wqo, finitely based, and has a rational generating function. Subclasses of $\operatorname{Geom}(M)$ are finite unions of geometric grid classes, and they also are wqo, finitely based and have rational generating functions.

Remarkably, geometric grid classes seem to arise naturally in structural descriptions of other pattern classes. Some early examples of this are in [6], and some quite advanced ones are in Murphy's thesis [51]. For example, Murphy shows that

$$
\operatorname{Av}(132,4312)=\text { Grid }\left(\begin{array}{rrr}
0 & 0 & 1 \\
1 & 0 & 0 \\
0 & 1 & 0 \\
0 & -1 & 0 \\
0 & 0 & -1
\end{array}\right) .
$$

It should be noted that these early 'applications' of geometric grid classes actually predate their formal introduction, but nonetheless essentially rely on their structural and enumerative properties.

In [5], Albert, Ruškuc and Vatter use geometric grid classes to obtain a general result on small classes, defined in terms of growth rates. The growth rate for a pattern class $C$ is defined as $\lim \sup _{n \rightarrow \infty} \sqrt[n]{\left|C_{n}\right| \text {, which }}$ exists by Markus-Tardos Theorem [50]. It is conjectured that the sequence $\sqrt[n]{\left|C_{n}\right|}$ actually always converges, but this is still open. In an earlier work, Vatter [62] showed that there are only countably many pattern classes with growth rate less than $\kappa$, and uncountably many of growth rate equal to $\kappa$, where $\kappa=2.20557 \ldots$ is the unique positive root of $1+2 x^{2}-x^{3}$. Incidentally the number $\kappa$ is related to the antichain $\mathcal{A}$ of Figure 4: it is the growth rate for the smallest class containing $\mathcal{A}$. This reinforces the notion of the antichain $\mathcal{A}$ being in a sense the smallest antichain in the permutation poset. Now, Theorem 5.3 says that geometric grid classes 
are strongly rational in the sense of [4], meaning that all their subclasses have rational generating functions. In [5] it is proved that for a geometric grid class $C$ and a strongly rational class $D$, the inflation $C[D]$ is again strongly rational. It is also proved that every pattern class of growth rate less than $\kappa$ is contained in an iterated inflation of the form $C[C[\ldots[C] \ldots]]$ for some geometric grid class. Combining all this together yields:

Theorem 5.4 (Albert, Ruškuc, Vatter [5]) Each of countably many pattern classes of permutation with growth rate less than $\kappa$ has a rational generating function.

Another general application of geometric grid classes exhibited in [5] is to wreath closures: it is shown that the wreath closure of a geometric grid class always has an algebraic generating function. Context free languages and grammars (as opposed to the regular ones for geometric grid classes) make an appearance in this proof, but outlining it is beyond the scope of the present survey.

It is perhaps interesting to point out that, somewhat unexpectedly, the decidability questions one may naturally ask about geometric grid classes seem not to have the obvious positive answers, which is certainly at variance with the situation for words described in Section 3. At present, it is not known how to compute the generating function or the basis from the gridding matrix, and it is also not known how to decide if a finitely based class is in fact a geometric grid class or at least contained in such a class. This is basically due to the non-constructive nature of appeals to Higman's Theorem in the argument. In a rare example of full grid classes behaving better than their geometric subclasses, Huczynska and Vatter [11] give a very nice constructive criterion for a finitely based class to be contained in a grid class. A different example of encoding permutations in a class by a regular language, in order to prove rationality of the generating function, can be found in [3]. It should be noted that the encoding there is not order preserving, and so an appeal to Higman's Theorem is not possible, and indeed the classes obtained are not all wqo.

It is perhaps disappointing that, despite the wealth of wqo results in other areas of combinatorics, there are very few results taking this further into structural, enumeration or computability corollaries. One exception are Petkovšek's letter graphs [56]. They are in a way similar to geometric grid classes, in that they rely on a finite specification (the role played by the matrix for permutations) under which every word over $A$ defines a graph. It is proved that the set of all graphs arising in this way from a fixed finite alphabet is wqo and, as a consequence, that it is decidable in polynomial time whether a graph is of this type. 


\section{Homomorphisms: embeddings and epimorphisms}

We have observed in Section 2 that the viewpoint of homomorphisms is a very natural one to take when describing orders on combinatorial structures. Embedding, perhaps the most frequently-encountered order in combinatorics, corresponds to the existence of an injective homomorphism, and the induced substructure ordering corresponds to the existence of a strong injective homomorphism. More complicated orders introduced into the theory, such as graph minor order, still admit natural simple conceptual descriptions via homomorphisms. It is therefore natural to ask wqo type questions for orders defined in terms of the existence of any homomorphism, or of a surjective homomorphism.

It is perhaps rather surprising that there seems to be very little evidence of such investigations in the literature. The homomorphism order itself has been studied, but the kind of questions we have been exploring here have received surprisingly little attention. As for the homomorphic image order, it has been almost entirely unexplored until a recent paper of the current authors ([34]).

The homomorphism order is defined by: $G \leq H$ if there exists a homomorphism from $G$ to $H$ (see [28]). Much, though not all, of the literature on orders defined via homomorphism, centres around graphs. This ordering is in fact a genuine quasi-order (i.e. it is not necessarily anti-symmetric) even if one restricts one's attention only to finite structures. For example for any two empty graphs $E_{m}$ and $E_{n}$ we have $E_{m} \leq E_{n} \leq E_{m}$. As with any quasi-order, $\leq$ induces a natural equivalence relation: $G$ and $H$ are defined to be homomorphically equivalent if $G \leq H \leq G$. Each equivalence class has a unique representative (up to isomorphism) of minimal size, called the core. We denote the resulting poset by $\mathcal{G}$; its elements are equivalence classes under the homomorphic equivalence, and the ordering is induced by the homomorphism ordering.

It transpires that this order is radically different from those we have encountered so far. This is probably best encapsulated in the following result:

Theorem 6.1 ([33], [28, Section 3.1]) The partial order $\mathcal{G}$ is universal, in the sense that it contains every countable partial order as a suborder.

This has immediate relevant consequences. For example, the poset $\mathcal{G}$ is not even well-founded, in contrast to all the other partial orders we have in this paper. Also, it contains a wealth of antichains. In particular, this poset is very far from being wqo. The significance of these antichains is 
further reduced by the fact that in the absence of well-foundedness not all ideals are defined as avoidance classes. Perhaps as a consequence, there has been only a limited study of antichains in this poset. It is known (Corollary 3.11, [28]) that there are only two finite maximal antichains, both of size one, in $\mathcal{G}$. The study of infinite antichains in $\mathcal{G}$ has centred around the notion of splitting (see for example [21]). The homomorphism order has been shown to be universal on various other classes of structures: digraphs ([58], oriented paths and trees ([33]), partial orders and lattices ([47]). Locally constrained graph homomorphisms - i.e. homomorphisms with the additional requirement that they restrict to bijections, injections or surjections on the neighbourhood of any vertex - have recently been explored in [24]. Interestingly, these restrictions are sufficient to bring us back from genuine quasi-orders into the world of partial orders.

Since the special case of injective homomorphisms has been so wellstudied, it would be natural to expect the embedding order's natural dual, the homomorphic image order, to have been similarly investigated. However, this is not the case. A notable exception is a 1979 paper [44] by Landraitis which considers the homomorphic image order on the set of countable linear order types. The author proves that this is a wqo, which mirrors an earlier result by Laver [46], asserting that the embedding relation is a wqo on this set. In fact, both authors prove that the orders under consideration are better quasi-orders.

In [34] the present authors attempt to redress this balance, by focussing on the homomorphic image order in the combinatorial setting, and considering the properties of this order in the realms of digraphs, graphs, words, permutations, posets, and a range of other combinatorial objects. In fact, as outlined in Section 2, we are led to define three homomorphic image orders, corresponding to the three flavours of homomorphism: standard, strong (or induced) and M-strong; let us denote these orderings as $\leq_{\mathrm{H}}$, $\leq_{\mathrm{IH}}, \leq_{\mathrm{MH}}$ respectively. In addition to antichains and wqo property, the paper contains discussions of the joint preimage property and the dual amalgamation property, which are natural analogues of the joint embedding property and amalgamation property in the embedding ordering.

It transpires that, unsurprisingly, under the M-strong homomorphic image order $\leq_{\mathrm{MH}}$, none of the classes of structures we consider are wqo. For the other two orderings the situation is more interesting. For digraphs, the collection of linear tournaments is an antichain under $\leq_{\mathrm{H}}$ (and hence $\leq_{\text {IH }}$ as well), while the collection of graphs $G_{n}$ illustrated in Figure 9 is an antichain under $\leq_{\mathrm{IH}}$, but not under $\leq_{\mathrm{H}}$. For graphs in the irreflexive representation the collection of all complete graphs $K_{n}$ forms an obvious antichain under $\leq_{\mathrm{H}}$. In the reflexive representation one can obtain an antichain by taking $K_{2 n}$ and removing the edges $\{2 i-1,2 i\}(i=1, \ldots, n)$. 


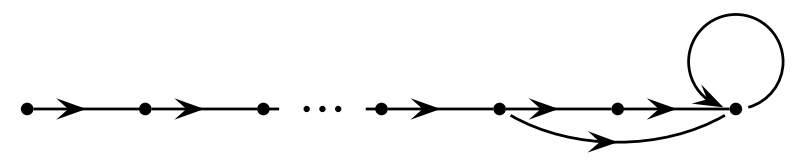

Figure 9: An antichain of digraphs under $\leq_{\mathrm{IH}}$ but not under $\leq_{\mathrm{H}}$

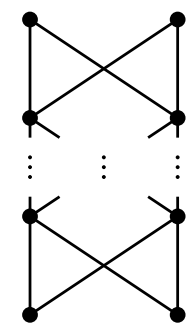

Figure 10: An antichain of posets under $\leq_{\mathrm{H}}$

A construction of a collection of reflexive graphs that would be an antichain under $\leq_{\mathrm{IH}}$ but not under $\leq_{\mathrm{H}}$ appears to be harder. For tournaments, the family $T_{n}$ with vertices $\{1, \ldots, n\}$ and edges

$$
\begin{array}{llll}
i \rightarrow j & \text { if } & i \neq \equiv j & (\bmod 2) \\
j \rightarrow i & \text { if } & i \equiv j & (\bmod 2)
\end{array}
$$

for $1 \leq i<j \leq n$, is an antichain under $\leq_{\mathrm{H}}$ in both the reflexive and irreflexive representations. The collection of posets is not wqo, in either the reflexive or non-reflexive representations, as witnessed for example by the family illustrated in Figure 10. All this leaves trees as yet again the boundary class, and here we prove:

Theorem 6.2 (Huczynska, Ruškuc [34]) The collection of all (reflexive or irreflexive) trees is well quasi-ordered by the homomorphic image ordering (and hence under the strong homomorphic image ordering as well).

Of the remaining structures, the homomorphic image ordering on permutations is a weakening of the subpermutation involvement, and so is not wqo. Likewise, the ordering on words is a weakening of the subword ordering, to the extent that wqo fails, as witnessed by the collection $(a b)^{n}$, 
$n=1,2, \ldots$. By way of contrast, the set of all finite equivalence relations is wqo under both $\leq_{\mathrm{H}}$ and $\leq_{\mathrm{IH}}$. This is proved by a relatively easy appeal to Higman's Theorem, mirroring the obvious proof that the equivalence relations are wqo under the embedding ordering.

Possible future research directions arising from this work are two-fold. On one hand, one can try to develop an analogue of the existing theory for substructure orderings, asking the equivalent questions in this new setting. Especially worthwhile in this direction would be a study of wqo for classes defined by avoidance conditions, with the aim of obtaining analogues to those previously-discussed for tournaments under subgraph order, or for graphs under topological minor order. On the other hand, motivated by the viewpoint outlined in this paper, it seems to us that a natural new direction is to investigate possible ways of combining the homomorphic image orderings and embedding orderings to produce a richer scheme of orderings for combinatorial structures. This would be guided by the observation that the minor ordering is a composition of the subgraph ordering and a special kind of a homomorphic image ordering (with all fibres being connected). In the presence of such a scheme, one would expect at the weak end of the spectrum not to have wqo, at the strong for it to be present, and then one could ask at which point (e.g. after how many compositions) it first occurs. One very concrete question that has occurred to us while writing the article is what happens if one strengthens one term in the above composition for the minor ordering and weakens the other. Specifically: For graphs $G$ and $H$, let $G \leq H$ if $G$ is a strong homomorphic image of an induced subgraph of $H$. Is the resulting ordering wqo?

\section{Conclusion}

We have seen that the property of being well quasi-ordered has been studied in a number of combinatorial settings for over half a century. The greatest body of results is within graph theory, where a variety of classes and orderings have been considered. The literature on wqo in the theory of pattern classes of permutations, while less well developed, contains some promising links with other combinatorial themes such as enumeration and asymptotics. It would appear that the language of homomorphisms, and in particular the interplay between embeddings and epimorphisms, may offer a conceptual link between different areas and methodologies, as well as a welcome extension of the field of investigation. 


\section{References}

[1] M.H. Albert and M.D. Atkinson, Simple permutations and pattern restricted permutations, Discrete Math. 300 (2005), 1-15.

[2] M.H. Albert, M.D. Atkinson, M. Bouvel, N. Ruškuc and V. Vatter, Geometric grid classes of permutations, Trans. Amer. Math. Soc. 365 (2013), 5859-5881.

[3] M.H. Albert, M.D. Atkinson and N. Ruškuc, Regular closed sets of permutations, Theoret. Comput. Sci. 306 (2003), 85-100.

[4] M.H. Albert, M.D. Atkinson and V. Vatter, Subclasses of the separable permutations, Bull. London Math. Soc. 5 (2011), 859-870.

[5] M.H. Albert, N. Ruškuc and V. Vatter, Inflations of geometric grid classes of permutations, Israel J. Math. (2014). DOI 10.1007/s11856014-1098-8.

[6] M.D. Atkinson, Restricted permutations, Discrete Math. 195 (1999), $27-38$.

[7] M.D. Atkinson, M.M. Murphy and N. Ruškuc, Partially well-ordered closed sets of permutations, Order 19 (2002), 101-113.

[8] F.M. Abu-Khzam and M.A. Langston, Graph coloring and the immersion order, in Computing and Combinatorics, T. Warnow and B. Zhu (eds.), Lecture Notes in Computer Science 2697, Springer, Berlin, 2003, pp. 394-403.

[9] M. Bousquet-Mélou, Algebraic generating functions in enumerative combinatorics and context-free languages, in STACS 2005, V. Diekert and B. Durand (eds.), Lecture Notes in Computer Science 3404, Springer, Berlin, 2005, pp. 18-35.

[10] R. Brignall, S. Huczynska and V. Vatter, Decomposing simple permutations, with enumerative consequences, Combinatorica 28 (2008), $385-400$.

[11] R. Brignall, N. Ruškuc and V. Vatter, Simple permutations: decidability and unavoidable substructures, Theoret. Comput. Sci. 391 (2008), 150-163.

[12] G. Cherlin, The Classification of Countable Homogeneous Directed Graphs and Countable Homogeneous n-Tournaments, Mem. Amer. Math. Soc. 131, AMS, Rhode Island, 1998. 
[13] G. Cherlin, Forbidden substructures and combinatorial dichotomies: WQO and universality, Discrete Math. 311 (2011), 1534-1584.

[14] M. Chudnovsky and P. Seymour, A well-quasi-order for tournaments, J. Combin. Theory, Ser. B 101 (2011), 47-53.

[15] D.G. Corneil, H. Lerchs and L.S. Burlingham, Complement reducible graphs, Discrete Appl. Math. 3 (1981), 163-174.

[16] J. Daligault, M. Rao and S. Thomassé, Well-quasi-order of relabel functions, Order 27 (2010), 301-315.

[17] P. Damaschke, Induced subgraphs and well quasi-ordering, J. Graph Theory 14 (1990), 427-435.

[18] R. Diestel, Graph Theory, Graduate Texts in Mathematics 173, Springer, Berlin, 2010

[19] G. Ding, Subgraphs and well quasi-ordering, J. Graph Theory 16 (1992), 489-502.

[20] G. Ding, Excluding a long double path minor, J. Combin. Theory, Ser. B 66 (1996), 11-23.

[21] D. Duffus, P.L. Erdös, J. Nešetřil and L. Soukup, Antichains in the homomorphism order of graphs, Comment. Math. Univ. Carolin. 48 (2007), 571-583.

[22] S. Eilenberg, Automata, Languages and Machines Vol A, Academic Press, NY, 1974.

[23] J. Fiala, J. Hubička and Y. Long, Universality of intervals of line graph order, European J. Combin. 41 (2014), 221-231.

[24] J. Fiala, D. Paulusma and J. A. Telle, Matrix and graph orders derived from locally constrained graph homomorphisms, in Mathematical Foundations of Computer Science 2005, Lecture Notes in Computer Science 3618, Springer Verlag, 2005, pp. 340-351.

[25] R. Fraïssé, Theory of Relations, North-Holland, Amsterdam, 1953.

[26] D. Glickenstein, Math 443/543 Graph Theory Notes 11, Department of Mathematics, University of Arizona, 2008.

[27] R. Govindana and S. Ramachandramurthi, A weak immersion relation on graphs and its applications, Discrete Math. 230 (2001), 189206. 
[28] P. Hell and J. Nešetřil, Graphs and Homomorphisms, Oxford Lecture Series in Mathematics and Its Applications 28, OUP, Oxford, 2004.

[29] C.W. Henson, Countable homogeneous relational systems and $N_{0^{-}}$ categorical theories, J. Symbolic Logic 37 (1972), 494-500.

[30] G. Higman, Ordering by divisibility in abstract algebras, Proc. London Math. Soc. 2 (1952), 326-336.

[31] W. Hodges, Model Theory, Cambridge University Press, Cambridge, 1993.

[32] J.E. Hopcroft and J.D. Ullman, Introduction to Automata Theory, Languages, and Computation, Addison-Wesley, Reading MA, 1979.

[33] J. Hubička and J. Nešetřil, Universal partial order represented by means of oriented trees and other simple graphs, European J. Combin. 26 (2005), 765-778.

[34] S. Huczynska and N. Ruškuc, Homomorphic image orders on combinatorial structures, Order (2014), to appear.

[35] I. Kim, On Containment Relations in Directed Graphs, PhD thesis, Princeton, 2013.

[36] N. Korpelainen and V. Lozin, Bipartite induced subgraphs and well quasi-ordering, J. Graph Theory 67 (2011), 235-249.

[37] N. Korpelainen and V. Lozin, Two forbidden induced subgraphs and well quasi-ordering, Discrete Math. 311 (2011), 1813-1822.

[38] N. Korpelainen, V. Lozin and I. Razgon, Boundary properties of well quasi-ordered sets of graphs, Order 30 (2013), 723-735.

[39] I. Kříž and J. Sgall, Well-quasi-ordering depends on the labels, Acta Sci. Math. 55 (1991), 59-65.

[40] I. Kříž and R. Thomas, On well-quasi-ordering finite structures with labels, Graphs Combin. 6 (1990), 41-49.

[41] J.B. Kruskal, Well-quasi-ordering, the tree theorem and Vaszsonyi's conjecture, Trans. Amer. Math. Soc. 95 (1960), 210-225.

[42] J.B. Kruskal, The theory of well quasi-ordering: a frequently discovered concept, J. Combin. Theory, Ser. A 13 (1972), 297-305. 
[43] K. Kuratowski, Sur le probléme des courbes gauches en topologie, Fund. Math. 15 (1930), 271-283.

[44] C. Landraitis, A combinatorial property of the homomorphism relation between countable order types, J. Symbolic Logic 44 (1979), 403-411.

[45] B. Latka, Finitely constrained classes of homogeneous directed graphs, J. Symbolic Logic 59 (1994), 124-139.

[46] R. Laver, On Fraïssé's order type conjecture, Ann. Math. (2) 93 (1971), 89-111.

[47] E. Lehtonen, Labeled posets are universal, European J. Combin. 29 (2008), 493-506.

[48] C-H. Liu, Graph Structures and Well Quasi-Ordering, PhD Thesis, Georgia Institute of Technology, 2014.

[49] W. Mader, Wohlquasigeordnete Klassen endlicher Graphen, J. Combin. Theory, Ser. B 12 (1972), 105-122.

[50] A. Marcus and G. Tardos, Excluded permutation matrices and the Stanley-Wilf conjecture, J. Combin. Theory, Ser. A 107 (2004), 153160 .

[51] M.M. Murphy, Restricted Permutations, Antichains, Atomic Classes and Stack Sorting, Ph.D. Thesis, University of St Andrews, 2003.

[52] M.M. Murphy and V. Vatter, Profile classes and partial well-order for permutations, Electron. J. Combin. 9 (2003), R17.

[53] C.St.J.A. Nash-Williams, On well-quasi-ordering finite trees. Math. Proc. Cambridge Philos. Soc. 59 (1963), 833-835.

[54] C.St.J.A. Nash-Williams, On well-quasi-ordering infinite trees, Math. Proc. Cambridge Philos. Soc. 61 (1965), 697-720.

[55] J. Nešetřil and P. Ossona de Mendez, Sparsity. Graphs, Structures, and Algorithms, Algorithms and Combinatorics 28, Springer, Heidelberg, 2012.

[56] M. Petkovšek, Letter graphs and well-quasi-order by induced subgraphs, Discrete Math. 244 (2002), 375-388. 
[57] M. Pouzet, Un bel ordre dábritement et ses rapports avec les bornes d'une multirelation, C. R. Acad. Sci. Paris Ser AB 274 (1972), 16771680.

[58] A. Pultr and V. Trnková, Combinatorial, Algebraic, and Topological Representations of Groups, Semigroups, and Categories, NorthHolland Mathematical Library 22, North-Holland, Amsterdam, 1980.

[59] N. Robertson and P. Seymour, Graph minors. XX. Wagners conjecture, J. Combin. Theory, Ser.B 92 (2004), 325-357.

[60] N. Robertson and P. Seymour, Graph minors. XXIII. Nash-Williams' immersion conjecture, J. Combin. Theory, Ser. B 100 (2010), 181205.

[61] R.P. Stanley, Enumerative Combinatorics Vol 2, Cambridge Studies in Advanced Mathematics 62, CUP, Cambridge, 1999.

[62] V. Vatter, Small permutation classes, Proc. London Math. Soc. 103 (2011), 879-921.

[63] K. Wagner, Über eine Eigenschaft der ebenen Komplexe, Math. Ann. 114 (1937), 570-590.

School of Mathematics and Statistics University of St Andrews St Andrews, U.K.

\{sophie.huczynska, nik.ruskuc\}@st-andrews.ac.uk 
